\title{
DOES ATORVASTATIN ALONE AMELIORATE CHRONIC PERIODONTITIS IN HYPERLIPIDEMIC PATIENTS? (A CROSS-SECTIONAL STUDY)
}

\author{
Hesham M. El-Sharkawy *, Nayef H. Felemban** and Bhari S. Manjunatha ***
}

\begin{abstract}
The present study was conducted to assess whether atorvastatin (ATV) alone could improve periodontal parameters and reduce inflammation and bone resorption in generalized chronic periodontitis $(\mathrm{gCP})$ patients with hyperlipidemia. One hundred and two subjects were classified into three groups: Group I included $34 \mathrm{gCP}$ patients with hyperlipidemia and maintained on a daily dose of $20 \mathrm{mg}$ atorvastatin at least for 6 months. Group II comprised $34 \mathrm{gCP}$ patients with hyperlipidemia and not taking any hypolipidemic drugs. Group III consisted of 34 normolipidemic individuals with $\mathrm{gCP}$. The periodontal parameters including plaque index (PI), bleeding on probing $(\mathrm{BOP})$, gingival index $(\mathrm{GI})$, probing depth $(\mathrm{PD})$ and clinical attachment loss $(\mathrm{CAL})$ were recorded and compared. Unstimulated whole saliva and blood samples were harvested from all subjects. Salivary levels of interleukin-1beta (IL-1 $\beta$ ) and receptor activator of nuclear kappa B ligand (RANKL) were evaluated by enzyme-linked immunosorbent assay (ELISA) technique. The blood samples were used to assess the lipid profile for all patients including total cholesterol (TC), triglycerides (TG), low-density lipoprotein-cholesterol (LDL-C) and high-density lipoproteincholesterol (HDL-C). The results demonstrated significantly greater decrease of periodontal parameters and salivary IL-1 $\beta$ and RANKL levels in group I compared to other groups. BOP, PD and CAL were positively correlated with the levels of salivary IL-1 $\beta$, TC, TG and LDL-C whereas HDL-C measurements were negatively associated with BOP, PD and CAL. In conclusion, ATV could be a viable therapeutic modality in $\mathrm{gCP}$ management, due to its anti-inflammatory and antiosteopathic effects. Salivary IL-1 $\beta$ and RANKL could serve as reliable biomarkers in periodontal disease.
\end{abstract}

KEYWORDS: Atorvastatin, Chronic periodontitis,, IL-1 $\beta$, RANKL, Lipid profile.

* Associate Professor of Oral Medicine and Periodontology, Faculty of Dentistry, Mansoura University, Mansoura, Egypt. Associate Professor of Oral Medicine and Periodontology, Faculty of Dentistry, Taif University, Taif, Kingdom of Saudi Arabia.

** Assistant Professor of Preventive Dentistry, Orthodontics Division, Faculty of Dentistry, Taif University, Taif, Kingdom of Saudi Arabia.

*** Associate professor of Oral Biology, Faculty of Dentistry, Taif University, Taif, Kingdom of Saudi Arabia. 


\section{INTRODUCTION}

In a recent consensus report, chronic periodontitis (CP) has been defined as a chronic multifactorial inflammatory disease affecting the tooth supporting apparatus. ${ }^{(1)} \mathrm{CP}$ is initiated by certain anaerobic bacteria and characterized by progressive destruction of the periodontal tissues leading eventually to tooth loss. ${ }^{(2)}$ It can also be classified among important global health problems because it affects the quality of life as it leads to tooth loss, causes masticatory dysfunction, deteriorates aesthetics, and impacts general systemic health. ${ }^{(3)}$ The majority of periodontal tissue destruction results from an exaggerated immunoinflammatory host response to periodontopathogenic bacteria and not by their direct effect. (2) The host response to these pathogens results in the release of a vast array of pro-inflammatory cytokines including tumor necrosis factor- $\alpha$ (TNF- $\alpha$ ), interleukin-1beta (IL-1 $\beta$ ) and prostaglandin-E2 (PGE2). ${ }^{(4)}$ These cytokines can activate a bunch of other mediators which lead to matrix degradation of the periodontium, and induce bone resorption by stimulating differentiation of new osteoclasts. ${ }^{(5)}$

Few decades ago, several drugs such as nonsteroidal anti-inflammatory drugs (NSAIDs), tetracyclines, and bisphosphonates have been assessed as host-modulatory therapeutics in the treatment of periodontitis.(6) Unfortunately, NSAIDs, used either systemically or topically, did not yield significant clinical benefits and have serious adverse effects. ${ }^{(7)}$ Tetracyclines demonstrated acceptable results when combined with non-surgical periodontal therapy; however, a variety of potential adverse events accompany tetracycline therapy, particularly photosensitivity. ${ }^{(8)}$ However, bisphosphonates were found to provide some improvements in periodontal parameters, their long term use was recently questioned due to the increased risk of osteonecrosis of the jaws after exodontia. ${ }^{(9)}$ Thus, periodontists and researchers all over the world are continually seeking for new host modulatory therapeutics to be used as adjunctive treatments for periodontal disease..$^{(2)}$

Recent investigations have revealed that patients suffering from periodontal disease have elevated serum levels of total cholesterol (TC), low density lipoprotein cholesterol (LDL-C), and triglycerides (TG), when compared to individuals with healthy periodontium..$^{(10)}$ On the other hand, hyperlipidemic subjects have shown significant incidence of periodontal disease in comparison to normolipidemic control individuals. Thus, a bidirectional relationship is likely to be found between periodontal disease and hyperlipidemia. ${ }^{(11)}$

Nowadays, atorvastatin (ATV) is considered one of the most commonly used hypolipidemic drugs that belong to the statins family. ${ }^{(12)}$ Statins are inhibitors of 3-hydroxy-3-methylglutaryl coenzyme A reductase (HMG-CoA reductase) which competitively and reversibly inhibit the mevalonate formation, which is the precursor of cholesterol synthesis. ${ }^{(13)}$ Statins are now known by their efficacy in reducing the serum cholesterol levels, safety and great tolerability. ${ }^{(14)}$ It was proved that statins reduce the serum levels of low density lipoprotein cholesterol (LDL-C). ${ }^{(15)}$ Furthermore, various investigators presented a strong convincing evidence that statins also have anti-inflammatory effect, in addition to their lipid-lowering properties. $^{(16)}$ More importantly, statins possess several potential pleiotropic effects such as antioxidant, immunomodulatory, and antithrombotic effects. $^{(17,18)}$ They also promote angiogenesis, ${ }^{(19)}$ enhance osteoblastic differentiation, ${ }^{(20)}$ and inhibit osteoclast formation. ${ }^{(21)}$ Therefore, the clinical benefits obtained from use of statins are greater than estimated.

To-date, an increased interest in saliva as a diagnostic biologic fluid has emerged among researchers. ${ }^{(22)}$ In the context of periodontal disease, 
unstimulated whole saliva is reported to reflect the soluble mediator composition of the gingival tissues, gingival crevicular fluid, and saliva in patients with periodontal disease. ${ }^{(23)}$ Potential biomarkers of periodontal disease were previously identified in saliva and shown to be specific for the unique physiologic and pathologic aspects of periodontitis. ${ }^{(24)}$ Changes in the concentrations of these biomarkers have diagnostic and therapeutic significance. ${ }^{(25)}$ For instance, salivary levels of interleukin-1beta (IL-1 $\beta$ ) were shown to be elevated in periodontitis patients and correlated positively to the clinical parameters, including bleeding on probing (BOP), pocket depth (PD) and clinical attachment loss (CAL). ${ }^{(26)}$

Recently, the receptor activator of nuclear factor kappa B ligand (RANKL) was linked with periodontal tissue destruction and bone remodeling. ${ }^{(27)}$ When it binds to its receptor RANK found on the surface of osteoclast precursors, generation of new osteoclasts occur. ${ }^{(28)}$ Moreover, binding of RANKL to RANK on mature osteoclasts enhances their adherence to bone surfaces and suppresses osteoclast apoptosis. ${ }^{(29)}$ Bostanci et al. reported that the concentration of RANKL in gingival crevicular fluid (GCF) is significantly increased in association with periodontal disease. ${ }^{(30)}$ Hence, we hypothesized that atorvastatin with its anti-inflammatory and antiosteopathic properties could improve the periodontal parameters and reduce the salivary levels of IL-1 $\beta$ and RANKL in gCP patients with hyperlipidemia who are controlled with atorvastatin.

Therefore, the purpose of this study was to assess whether atorvastatin alone could improve periodontal parameters and resolve inflammation and bone resorption in hyperlipidemic patients with gCP.

\section{PATIENTS AND METHODS}

\section{Study Population}

A total of 154 hyperlipidemic individuals and 87 normolipidemic subjects were recruited from King Abdulaziz general hospital in Taif city, Saudi Arabia to the Periodontics clinic, Faculty of Dentistry at Taif University between January and May, 2017 for screening and selection of eligible subjects in order to participate in the present study. The inclusion criteria included all subjects who had moderate to severe generalized chronic periodontitis $(\mathrm{gCP})$ based on Armitage classification (31) with an age range from 40-60 years old. Eligible individuals were non-smokers and had no history of any systemic diseases such as diabetes mellitus, hypertension, autoimmune diseases and cancer. The selected participants did not receive any previous treatment for $\mathrm{gCP}$ at least in the last 6 months, did not take any antibiotics or non-steroidal anti-inflammatory drugs for 3 months before commencement of the study. The enrolled patients were classified into three groups:

Group I (CP+ATV) which included $34 \mathrm{gCP}$ patients with hyperlipidemia and maintained on a daily dose of $20 \mathrm{mg}$ atorvastatin at least for 6 months.

Group II (CPH), comprised $34 \mathrm{gCP}$ patients with hyperlipidemia and not taking any hypolipidemic drugs.

Group III (CPN) consisted of 34 normolipidemic individuals with $\mathrm{gCP}$.

All study participants signed informed consents. The study was approved by the Institutional Review Board (IRB) of Taif University.

\section{Periodontal Assessment}

The following clinical periodontal indices were assessed in study groups: the plaque index (PI) ${ }^{(32)}$, bleeding on probing (BOP) recorded as percentage, gingival index (GI) ${ }^{(33)}$, probing depth (PD) and clinical attachment loss (CAL). 


\section{Saliva Collection}

Saliva samples were collected in the morning between 8 to 10 a.m. from all participants after fasting overnight. All participants were asked not to take any drinks except water after getting up. Whole unstimulated saliva samples were harvested by expectoration into $5 \mathrm{~mL}$ sterile polypropylene tubes before recording the clinical measurements. All saliva samples were centrifuged to remove debris, and immediately frozen and stored at $-80^{\circ} \mathrm{C}$ until evaluation time.

\section{Biochemical Assessment}

Immediately after saliva collection, $4 \mathrm{~mL}$ of venous blood samples were withdrawn from the antecubital fossa of the arm under complete aseptic condition from all patients. The blood samples were left to clot for 20 minutes. Then blood samples were centrifuged at $3000 \mathrm{rpm}$ for 10 minutes. Clear sera were separated and used for lipid profile analysis which includes total cholesterol (TC), triglycerides (TG) and HDL-C, using specific kits supplied by SPINREACT (Sant Esteve de bas (GI), Spain). Low-density lipoprotein-cholesterol (LDL-C) was calculated by using Friedewald equation: LDL$\mathrm{C}=$ Total cholesterol- HDL-TG/5 (mg/dL). ${ }^{(34)}$

The two important aspects of periodontal disease, inflammation and bone destruction, were evaluated in the patient population by determining the salivary levels of IL-1 $\beta$; an important mediator of tissue destruction in inflammatory diseases, and RANKL; that promotes bone resorption through the activation of osteoclasts. Both analytes were determined in saliva by enzyme-linked immunosorbent assay (ELISA). Saliva samples were pipetted into clean microcap tubes and centrifuged at $10,000 \times \mathrm{g}$ for 5 minutes. Then, the supernatants were transferred to clean microcap tubes and used immediately for ELISA assay. Human-RANKL and IL-1 $\beta$ ELISA development kits (R\&D systems, Abingdon Science Park Abingdon, UK) were used to quantify these molecules in saliva samples according to the manufacturers' recommendations. The results of salivary RANKL and IL-1 $\beta$ assays are expressed as concentrations in picograms per milliliter.

\section{Statistical Analysis of Data}

Data were analyzed using IBM SPSS software package version 19. The mean and standard deviation were used to describe quantitative data after testing normality via Kolmogorov-Smirnov test. The ANOVA test was used to compare between normally distributed data of the three study groups. Moreover, Pearson's correlation test was used to assess the correlation between each two normally distributed quantitative variables. The significance of the yielded results was considered at $5 \%$ value.

\section{RESULTS}

A total of 68 out of 154 hyperlipidemic individuals suffering from $\mathrm{gCP}$ were selected for participation in the study; 34 participants (group I; CP+ATV group) were maintained on a daily dose of $20 \mathrm{mg}$ atorvastatin for a minimum period of 6 months with an age range 41-58 years and female participants represented $50 \%$ in this group. The other 34 individuals who were not taking any drugs for hypercholesterolemia represented group II (CPH group) with an age range 43-57 years and females constituted $47 \%$ in the group. A third group (CPN group) included 34 out of 87 normolipidemic individuals with gCP with an age range 40-55 years. Female patients constituted 55\% in CPN group.

The demographics, clinical and lipid profile features of the study groups were indicated in table 1. There were no statistically significant variations among groups regarding age, sex and number of existing teeth. In group I (CP+ATV group), the average duration of atorvastatin intake was $16.8 \pm 3.4$ months, whereas the value was zero for the other groups, $\mathrm{p}=0$.

Table 1 also shows the lipid profile data of all groups. Group II (CPN) showed significant differences with comparison to group I and III 
regarding the levels of total cholesterol (TC), triglycerides (TG), HDL-C and LDL-C ( $\mathrm{p}<0.001)$. However, there were no statistically significant differences between group I and III regarding the afore-mentioned biochemical analytes $(\mathrm{p}>0.05)$.

Table 2 demonstrates the mean values $( \pm \mathrm{SD})$ of plaque index (PI), gingival index (GI), bleeding on probing percentage (BOP) as percentage, probing depth (PD) and clinical attachment loss (CAL) of all study groups. The PI values showed nonsignificant differences among groups, $\mathrm{p}>0.05$.
However, the mean GI values of group I, II and III were $0.9 \pm 0.3,1.5 \pm 0.2$ and $1.3 \pm 0.4$; respectively. Statistically, a significant variation of GI values of group I was noted compared to other groups, $\mathrm{p}$ $<0.01$. In a similar pattern, BOP in group I showed a statistically significant variation when compared to group II and III, p <0.01. More importantly, PD and CAL measurements of group I showed statistically significant differences in comparison to group II and III, $\mathrm{p}<0.01$. In addition, group II had no statistically significant differences of GI, BOP, PD and CAL values when compared to group III, $\mathrm{p}>0.05$.

TABLE (1) Patients demographics, clinical and lipid profile features

\begin{tabular}{|c|c|c|c|c|}
\hline Variable & $\begin{array}{c}\text { Group I } \\
\begin{array}{c}\text { (CP+ATV) } \\
\text { n=34 }\end{array}\end{array}$ & $\begin{array}{c}\text { Group II } \\
\begin{array}{c}\text { (CPH) } \\
\text { n=34 }\end{array}\end{array}$ & $\begin{array}{c}\text { Group III } \\
\begin{array}{c}\text { (CPN) } \\
\text { n=34 }\end{array}\end{array}$ & p-value \\
\hline Age (years) & $48.2 \pm 4.7$ & $47.9 \pm 5.1$ & $45.3 \pm 7.2$ & NS \\
\hline Age range (minimum-maximum) & $41-58$ & $43-57$ & $40-55$ & NS \\
\hline Female, n (\%) & $17(50)$ & $16(47)$ & $19(55)$ & NS \\
\hline Teeth (n) & $19.2 \pm 3.7$ & $21.3 \pm 2.9$ & $20.7 \pm 2.6$ & $\mathrm{NS}$ \\
\hline Duration of atorvastatin intake (months) & $16.8 \pm 3.4 *$ & 0 & 0 & 0 \\
\hline Total Cholesterol (TC) (mg/dL) & $182.3 \pm 9.2$ & $231.4 \pm 7.6^{*}$ & $174.8 \pm 6.1$ & $<0.001$ \\
\hline Triglycerides (TG) (mg/dL) & $141.4 \pm 9.3$ & $179.6 \pm 13.8 *$ & $136.2 \pm 11.7$ & $<0.001$ \\
\hline HDL-C (mg/dL) & $54.3 \pm 5.8$ & $37.4 \pm 4.1 *$ & $57.4 \pm 4.1$ & $<0.001$ \\
\hline LDL-C (mg/dL) & $76.2 \pm 9.5$ & $124.3 \pm 11.9^{*}$ & $69.2 \pm 7.8$ & $<0.001$ \\
\hline
\end{tabular}

$* p=A N O V A$ test $($ statistically significant when $p<0.05$ ).

NS denotes non-significant variation ( $p>0.05)$.

TABLE (2) The average values $( \pm \mathrm{SD}$ ) of full mouth periodontal indices of study groups

\begin{tabular}{|l|c|c|c|}
\hline Parameter & $\begin{array}{c}\text { Group I } \\
(\mathbf{C P}+\mathbf{A T V}) \\
\mathbf{n = 3 4}\end{array}$ & $\begin{array}{c}\text { Group II } \\
\text { (CPH) } \\
\mathbf{n = 3 4}\end{array}$ & $\begin{array}{c}\text { Group III } \\
\text { (CPN) } \\
\mathbf{n = 3 4}\end{array}$ \\
\hline PI & $1.9 \pm 0.3$ & $2.1 \pm 0.2$ & $2.2 \pm 0.1$ \\
\hline GI & $0.9 \pm 0.3^{*}$ & $1.5 \pm 0.2$ & $1.3 \pm 0.4$ \\
\hline BOP $(\%)$ & $42 \pm 19^{*}$ & $73 \pm 14$ & $67 \pm 17$ \\
\hline PD $(\mathbf{m m})$ & $3.89 \pm 0.73^{*}$ & $4.56 \pm 0.61$ & $4.39 \pm 0.48$ \\
\hline CAL $(\mathbf{m m})$ & $4.76 \pm 0.92^{*}$ & $5.16 \pm 0.54$ & $5.09 \pm 0.74$ \\
\hline
\end{tabular}

$* p=$ ANOVA test $(p<0.01)$. 
Table 3 displays the comparison between the average salivary IL-1 $\beta$ and salivary RANKL levels in study groups. The mean salivary IL-1 $\beta$ levels for groups I, II and III were $429 \pm 143,874 \pm 115$ and $649 \pm 97 \mathrm{pg} / \mathrm{mL}$; respectively. A highly significant variation was noted for salivary IL- $1 \beta$ levels in group I in comparison to the corresponding values of group II and III, $\mathrm{p}<0.001$. Moreover, there was a significant difference in salivary IL- $1 \beta$ levels in group II with regard to group III, $\mathrm{p}<0.01$. Of interest, it was noted that the average IL- $1 \beta$ level in group II are almost two- fold the corresponding value in group $\mathrm{I}$.

The mean salivary RANKL level in group I was $87 \pm 26 \mathrm{pg} / \mathrm{mL}$ whereas its mean value was $122 \pm 31$ and $115 \pm 23 \mathrm{pg} / \mathrm{mL}$ for group II and III; respectively. Similarly, a significant difference was noted for salivary RANKL levels in group I in comparison to groups II and III, $\mathrm{p}<0.001$. Of interest, it was observed that the average salivary RANKL level in group II was 1.4-fold the mean value of group I. However, there was no significant variation of salivary RANKL levels of group II compared to group III, $\mathrm{p}>0.05$.
In table 4 , the correlation matrix between clinical periodontal measurements and biochemical analytes calculated by Pearson's test was indicated. The values of GI were positively correlated only with the values of salivary IL- $1 \beta$. Moreover, the values of BOP were positively correlated with the values of salivary IL- $1 \beta$, TC, TG and LDL-C whereas BOP values were negatively associated with HDL-C values.

Furthermore, the values of PD and CAL were positively associated with the values of salivary IL-1 $\beta$, RANKL, TC, TG and LDL-C. On the other hand, the PD and CAL measurements were negatively correlated with the values of HDL-C.

TABLE (3) Salivary interleukin $1-\beta$ (IL-1 $\beta$ ) and salivary RANKL levels in study groups

\begin{tabular}{|c|c|c|c|}
\hline Parameter & $\begin{array}{c}\text { Group I } \\
(\mathbf{C P + A T V}) \\
\mathbf{n = 3 4}\end{array}$ & $\begin{array}{c}\text { Group II } \\
\text { (CPH) } \\
\mathbf{n = 3 4}\end{array}$ & $\begin{array}{c}\text { Group III } \\
(\mathbf{C P N}) \\
\mathbf{n = 3 4}\end{array}$ \\
\hline IL-1/ $(\mathbf{p g} / \mathbf{m L})$ & $429 \pm 143^{*}$ & $874 \pm 115$ & $649 \pm 97 * *$ \\
\hline RANKL $(\mathbf{p g} / \mathbf{m L})$ & $87 \pm 26^{*}$ & $122 \pm 31$ & $115 \pm 23$ \\
\hline
\end{tabular}

${ }^{*} p=$ ANOVA test $(p<0.001) . * * *=$ ANOVA test $(p<0.01)$.

TABLE (4) Correlation matrix of clinical periodontal parameters versus biochemical measurements

\begin{tabular}{|c|c|c|c|c|c|c|c|}
\hline Parameter & & IL-1 $\beta$ & RANKL & TC & TG & HDL-C & LDL-C \\
\hline \multirow{2}{*}{ PI } & $\mathrm{r}$ & 0.438 & 0.249 & 0.323 & 0.148 & -0.324 & 0.399 \\
\hline & $\mathrm{p}$ & 0.313 & 0.534 & 0.146 & 0.417 & 0.17 & 0.257 \\
\hline \multirow{2}{*}{ GI } & $\mathrm{r}$ & 0.584 & 0.371 & 0.226 & 0.329 & -0.398 & 0.494 \\
\hline & $\mathrm{p}$ & $0.027 *$ & 0.124 & 0.245 & 0.426 & 0.524 & 0.0912 \\
\hline \multirow{2}{*}{ BOP } & $\mathrm{r}$ & 0.579 & 0.409 & 0.646 & 0.702 & -0.697 & 0.591 \\
\hline & $\mathrm{p}$ & $0.037 *$ & 0.055 & $0.031 *$ & $0.019 *$ & $0.043 *$ & 0.034* \\
\hline \multirow{2}{*}{ PD } & $\mathrm{r}$ & 0.592 & 0.612 & 0.629 & 0.732 & -0.793 & 0.526 \\
\hline & $\mathrm{p}$ & $0.041 *$ & $0.013 *$ & $0.027 *$ & $0.024 *$ & $0.046^{*}$ & $0.029 *$ \\
\hline \multirow{2}{*}{ CAL } & $\mathrm{r}$ & 0.629 & 0.568 & 0.570 & 0.644 & -0.712 & 0.626 \\
\hline & $\mathrm{p}$ & $0.024 *$ & $0.035^{*}$ & $0.048 *$ & $0.027 *$ & 0.031* & $0.016 *$ \\
\hline
\end{tabular}

${ }^{*} p=$ statistically significant when $p<0.05$. 


\section{DISCUSSION}

In this cross-sectional study, the effectiveness of atorvastatin (ATV), which is a commonly used hypocholesterolemic agent, in improving generalized chronic periodontitis $(\mathrm{gCP})$ in patients with hyperlipidemia was addressed. ATV, as one of the statins family, has been extensively prescribed by physicians worldwide in order to prevent cardiovascular accidents. ${ }^{(35)}$ In addition to the hypolipidemic effect of statins , they were found to possess several pleiotropic actions, particularly, anti-inflammatory, immunomodulatory, antioxidant effects, as well as increasing osteoblastic differentiation and inhibiting bone resorption. ${ }^{(36)}$

Several previous investigations used statins either systemically or as a local delivery therapeutic in periodontal treatment showing promising results. $^{(37-40)}$ However, other researchers did not yield any additional benefits to periodontal therapy from statin use. ${ }^{(41)}$ Therefore, we designed this study to evaluate whether ATV alone has the potential to ameliorate periodontal indices, resolve inflammation and affect bone resorption in $\mathrm{gCP}$ patients with hyperlipidemia. Thus, in our study, we have tested the effect of ATV intake in hyperlipidemic patients with $\mathrm{gCP}(\mathrm{CP}+\mathrm{ATV}$ group) on the periodontal parameters and salivary levels of both IL-1 $\beta$ (as a potent inflammatory marker) and RANKL (as an osteoclast differentiation marker) compared to a group of hyperlipidemic subjects with $\mathrm{gCP}$ without intake of any hypolipidemic drugs ( $\mathrm{CPH}$ group) and another group of normolipidemic patients with $\mathrm{gCP}$ (CPN group). Our findings showed significantly greater reduction in GI, BOP, $\mathrm{PD}$ and $\mathrm{CAL}$ in the $\mathrm{CP}+\mathrm{ATV}$ group when compared to $\mathrm{CPH}$ and $\mathrm{CPN}$ groups. Moreover, the salivary levels of both IL$1 \beta$ and RANKL in the CP+ATV group decreased significantly in comparison to the other groups. These findings were consistent with the results of previous studies ${ }^{(42-44)}$ For instance, in another similar cross-sectional study, hyperlipidaemic patients who were not taking ATV, when compared with normolipidemic individuals and the hyperlipidemic group which used a daily dose of $20 \mathrm{mg}$ ATV, had significantly increased gingival bleeding index and probing depth. It was concluded that ATV as a statin has an appreciated effect on periodontal health status. ${ }^{(45)}$ However, the previous study did not test any biological markers to confirm their findings from the biochemical point of view as carried out in our study.

A cross-sectional study carried out by Meisel and his colleagues assessed the effects of statins on periodontal parameters. Their findings indicated that serum C-reactive protein (CRP) levels were elevated in peridontitis patients who did not use statins, however, in statin users with periodontal disease, CRP levels were significantly lower. ${ }^{(46)}$ These results came in accordance with our findings, but the authors used a different inflammatory mediator (CRP) than those investigated in our study.

In our study, we hypothesized that ATV might have the potential to decrease two cytokines in saliva, IL- $1 \beta$ representing one of the potent inflammatory periodontal cytokines as well as RANKL which is considered as a novel bone cytokine responsible for osteoclast differentiation and activity. ${ }^{(47)}$ We also decided to estimate the afore-mentioned cytokines in unstimulated saliva because collection of saliva is a non invasive method and easily harvested in large amount and reflects all soluble mediators and cytokines in the periodontal tissues as most of the gingival sulcular fluid seeps into saliva. ${ }^{(48)}$ To the best of our knowledge, there is no studies available until now investigating the salivary RANKL levels in chronic periodontitis patients who are maintained on a daily stable dose of statins. However, ElSharkawy et al, 2010, assessed salivary RANKL in periodontitis patients after supplementation with omega-3 fatty acids combined with low dose of aspirin and demonstrated greater reduction in salivary RANKL when compared to the non-omega-3 
group after 3 and 6 months of therapy. ${ }^{(49)}$ Salivary RANKL was also reduced in periodontal disease after topical application of melatonin on the gingiva in another study carried out by Cutando and his coworkers. ${ }^{(50)}$ Previously, Jin et al., 2014, reported that simvastatin can inhibit lipopolysaccharide-induced osteoclastogenesis and decreases alveolar bone loss in experimental periodontal disease. ${ }^{(51)}$

One of the interesting findings in our study is that the average salivary levels of IL- $1 \beta$ and RANKL in $\mathrm{CP}+\mathrm{ATV}$ group are nearly twice and 1.4 -fold the mean values of the corresponding measurements in $\mathrm{CPH}$ group; respectively.

Our study also demonstrated the presence of positive association of GI with the salivary levels of IL-1 $\beta$. The results of the present study also showed that BOP values were positively correlated with the values of salivary IL- $1 \beta$, TC, TG and LDL-C, however, BOP were negatively associated with the values of HDL-C. Importantly, the values of $\mathrm{PD}$ and CAL were positively associated with the values of salivary IL-1 $\beta$, RANKL, TC, TG and LDL-C whereas; the PD and CAL measurements were negatively associated with HDL-C values. This comes in agreement with the findings obtained by other investigators. For instance, Katz and co-workers confirmed a positive association between deep periodontal pockets and high TC and LDL levels, by using the index Community Periodontal Index of Treatment Needs (CPITN). ${ }^{(52)}$ Furthermore, a recent meta-analysis confirmed that chronic periodontitis has a significantly positive relation with increased serum TG and TC, ${ }^{(53)}$ which conforms with our findings.

The anti-inflammatory action of statins, which is essential for periodontal therapy, has been proved from the results of the present study. This can be explained as inhibition of isoprenoids by statins interferes with the inflammatory cell signaling pathways, hence, reducing pro-inflammatory cytokine expression. ${ }^{(54)}$
There is a strong evidence supporting a positive effect of statins on bone architecture. ${ }^{(55)}$ Alveolar crestal bone loss is a characteristic feature of $\mathrm{CP}$, and decreasing bone resorption rate is a crucial goal for periodontal therapy. ${ }^{(56)}$ The present study also has highlighted that salivary RANKL could be a potential marker for activity of bone resorption in $\mathrm{CP}$ patients as our results showed greater reduction of salivary RANKL in ATV users. Thus, statins have the potential to significantly inhibit alveolar bone loss in periodontal disease. ${ }^{(21)}$ While our data demonstrate reduction of the periodontal parameters and salivary levels of IL- $\beta$ and RANKL in ATV users, there are several important limitations of the present cross-sectional study. First, the sample size is not large enough to be generalizable to all users of different types of statins, so, larger longitudinal studies are needed in order to determine whether these results are generalizable to various statins users. Second, we used a cross-sectional study design which inherently does not give the best scientific evidence in evidence based dentistry as randomized clinical trials. ${ }^{(57)}$ It is of great importance to confirm our findings in future randomized clinical trials by using different types of statins.

\section{CONCLUSIONS}

Our findings suggest that ATV, in addition to its hypolipidemic effect, could be an effective therapeutic modality in the management of periodontal disease, due to its beneficial antiinflammatory and antiosteopathic properties. It was also concluded that salivary IL-1 $\beta$ and salivary RANKL are potential reliable markers for diagnosis and treatment of periodontal disease. BOP, PD and CAL are positively correlated with the levels of salivary IL-1 $\beta$, TC, TG and LDL-C whereas HDL-C measurements are negatively associated with BOP, PD and CAL. More clinical human studies of statins are warranted to elucidate its therapeutic potential in periodontal disease. 


\section{REFERENCES}

1. Bascones A, Noronha S, Gomez M, Mota P, Gonzalez Moles MA, Villarroel Dorrego M. Tissue destruction in periodontitis: bacteria or cytokines fault? Quintessence international 2005;36:299-306.

2. Salvi GE, Lang NP. Host response modulation in the management of periodontal diseases. Journal of clinical periodontology 2005;32 Suppl 6:108-129.

3. Naorungroj S, Slade GD, Divaris K, Heiss G, Offenbacher $\mathrm{S}$, Beck JD. Racial differences in periodontal disease and 10-year self-reported tooth loss among late middle-aged and older adults: the dental ARIC study. Journal of public health dentistry 2017.

4. Graves DT. The potential role of chemokines and inflammatory cytokines in periodontal disease progression. Clinical infectious diseases : an official publication of the Infectious Diseases Society of America 1999;28:482-490.

5. Ohmori Y. [Mechanisms of bone resorption in periodontal disease]. Clinical calcium 2001;11:302-308.

6. Williams RC. Host modulation for the treatment of periodontal disease. Compendium of continuing education in dentistry 2008;29:160-162, 164, 166-168 passim.

7. Heasman PA, Benn DK, Kelly PJ, Seymour RA, Aitken D. The use of topical flurbiprofen as an adjunct to nonsurgical management of periodontal disease. Journal of clinical periodontology 1993;20:457-464.

8. Fernandes LA, Martins TM, Almeida JM, et al. Experimental periodontal disease treatment by subgingival irrigation with tetracycline hydrochloride in rats. Journal of applied oral science : revista FOB 2010;18:635-640.

9. Aghaloo TL, Kang B, Sung EC, et al. Periodontal disease and bisphosphonates induce osteonecrosis of the jaws in the rat. Journal of bone and mineral research : the official journal of the American Society for Bone and Mineral Research 2011;26:1871-1882.

10. Machado AC, Quirino MR, Nascimento LF. Relation between chronic periodontal disease and plasmatic levels of triglycerides, total cholesterol and fractions. Brazilian oral research 2005;19:284-289.

11. Jaramillo A, Lafaurie GI, Millan LV, et al. Association between periodontal disease and plasma levels of cholesterol and triglycerides. Colombia medica 2013; 44:80-86.
12. McCrindle BW, Ose L, Marais AD. Efficacy and safety of atorvastatin in children and adolescents with familial hypercholesterolemia or severe hyperlipidemia: a multicenter, randomized, placebo-controlled trial. The Journal of pediatrics 2003;143:74-80.

13. Sirtori CR, Calabresi L, Pisciotta L, et al. Effect of statins on LDL particle size in patients with familial combined hyperlipidemia: a comparison between atorvastatin and pravastatin. Nutrition, metabolism, and cardiovascular diseases : NMCD 2005;15:47-55.

14. Shekhar Pandey A, Bissonnette S, Boukas S, Rampakakis E, Sampalis JS. Effectiveness and tolerability of ezetimibe co-administered with statins versus statin dose-doubling in high-risk patients with persistent hyperlipidemia: The EZE(STAT)2 trial. Archives of medical science : AMS 2011;7:767-775.

15. Liao JK. Effects of statins on 3-hydroxy-3-methylglutaryl coenzyme a reductase inhibition beyond low-density lipoprotein cholesterol. The American journal of cardiology 2005; 96:24F-33F

16. Quist-Paulsen P. Statins and inflammation: an update. Current opinion in cardiology 2010;25:399-405.

17. Yilmaz MI, Baykal Y, Kilic M, et al. Effects of statins on oxidative stress. Biological trace element research 2004;98:119-127.

18. Sexton T, Wallace EL, Smyth SS. Anti-Thrombotic Effects of Statins in Acute Coronary Syndromes: At the Intersection of Thrombosis, Inflammation, and PlateletLeukocyte Interactions. Current cardiology reviews 2016;12:324-329.

19. Weis M, Heeschen C, Glassford AJ, Cooke JP. Statins have biphasic effects on angiogenesis. Circulation 2002; 105:739-745.

20. Garrett IR, Mundy GR. The role of statins as potential targets for bone formation. Arthritis research 2002; 4:237-240.

21. de Mones E, Schlaubitz S, Catros S, Fricain JC. Statins and alveolar bone resorption: a narrative review of preclinical and clinical studies. Oral surgery, oral medicine, oral pathology and oral radiology 2015;119:65-73.

22. Zhang CZ, Cheng XQ, Li JY, et al. Saliva in the diagnosis of diseases. International journal of oral science 2016; 8:133-137.

23. Llena-Puy $\mathrm{C}$. The role of saliva in maintaining oral health and as an aid to diagnosis. Medicina oral, patologia oral y cirugia bucal 2006;11:E449-455. 
24. Brinkmann O, Zhang L, Giannobile WV, Wong DT. Salivary biomarkers for periodontal disease diagnostics. Expert opinion on medical diagnostics 2011;5:25-35.

25. Miller CS, King CP, Jr., Langub MC, Kryscio RJ, Thomas MV. Salivary biomarkers of existing periodontal disease: a cross-sectional study. Journal of the American Dental Association 2006;137:322-329.

26. Alshehri M, Alshehri FA, Alshail F. Effect of scaling and root planing with and without adjunct use of an essentialoil-based mouthwash on whole salivary interleukin-1beta levels in patients with periodontal disease: A short-term follow-up study. Interventional medicine \& applied science 2016;8:3-6.

27. Chen B, Wu W, Sun W, Zhang Q, Yan F, Xiao Y. RANKL expression in periodontal disease: where does RANKL come from? BioMed research international 2014;2014:731039.

28. Liu D, Xu JK, Figliomeni L, et al. Expression of RANKL and OPG mRNA in periodontal disease: possible involvement in bone destruction. International journal of molecular medicine 2003;11:17-21.

29. Sutherland KA, Rogers HL, Tosh D, Rogers MJ. RANKL increases the level of Mcl-1 in osteoclasts and reduces bisphosphonate-induced osteoclast apoptosis in vitro. Arthritis research \& therapy 2009;11:R58.

30. Bostanci N, Ilgenli T, Emingil G, et al. Gingival crevicular fluid levels of RANKL and OPG in periodontal diseases: implications of their relative ratio. Journal of clinical periodontology 2007;34:370-376.

31. Armitage GC. Development of a classification system for periodontal diseases and conditions. Northwest dentistry 2000;79:31-35.

32. Silness J, Loe H. Periodontal Disease in Pregnancy. Ii. Correlation between Oral Hygiene and Periodontal Condtion. Acta odontologica Scandinavica 1964;22:121135 .

33. Loe H, Silness J. Periodontal Disease in Pregnancy. I. Prevalence and Severity. Acta odontologica Scandinavica 1963;21:533-551.

34. Friedewald WT, Levy RI, Fredrickson DS. Estimation of the concentration of low-density lipoprotein cholesterol in plasma, without use of the preparative ultracentrifuge. Clinical chemistry 1972;18:499-502.

35. LaRosa JC, Grundy SM, Kastelein JJ, et al. Safety and efficacy of Atorvastatin-induced very low-density lipoprotein cholesterol levels in Patients with coronary heart disease (a post hoc analysis of the treating to new targets [TNT] study). The American journal of cardiology 2007;100:747-752.

36. Estanislau IM, Terceiro IR, Lisboa MR, et al. Pleiotropic effects of statins on the treatment of chronic periodontitis--a systematic review. British journal of clinical pharmacology 2015;79:877-885.

37. Pradeep AR, Thorat MS. Clinical effect of subgingivally delivered simvastatin in the treatment of patients with chronic periodontitis: a randomized clinical trial. Journal of periodontology 2010;81:214-222.

38. Rao NS, Pradeep AR, Bajaj P, Kumari M, Naik SB. Simvastatin local drug delivery in smokers with chronic periodontitis: a randomized controlled clinical trial. Australian dental journal 2013;58:156-162.

39. Subramanian S, Emami H, Vucic E, et al. High-dose atorvastatin reduces periodontal inflammation: a novel pleiotropic effect of statins. Journal of the American College of Cardiology 2013;62:2382-2391.

40. Fajardo ME, Rocha ML, Sanchez-Marin FJ, EspinosaChavez EJ. Effect of atorvastatin on chronic periodontitis: a randomized pilot study. Journal of clinical periodontology 2010;37:1016-1022.

41. Saxlin T, Suominen-Taipale L, Knuuttila M,Alha P, Ylostalo P. Dual effect of statin medication on the periodontium. Journal of clinical periodontology 2009;36:997-1003.

42. Sayar F, Fallah S, Akhondi N, Jamshidi S. Association of serum lipid indices and statin consumption with periodontal status. Oral diseases 2016;22:775-780.

43. Shivakumar T, Patil VA, Desai MH. Periodontal status in subjects with hyperlipidemia and determination of association between hyperlipidemia and periodontal health: a clinicobiochemical study. The journal of contemporary dental practice 2013;14:785-789.

44. Lee S, Im A, Burm E, Ha M. Association Between Periodontitis With Blood Lipid Levels in Korean Population. Journal of periodontology 2017:1-10.

45. Sangwan A, Tewari S, Singh H, Sharma RK, Narula SC. Periodontal status and hyperlipidemia: statin users versus non-users. Journal of periodontology 2013;84:3-12.

46. Meisel P, Kohlmann T, Wallaschofski H, Kroemer HK, Kocher T. Cholesterol, C-Reactive Protein, and Periodontitis: HMG-CoA-Reductase Inhibitors (Statins) as Effect Modifiers. ISRN dentistry 2011;2011:125168. 
47. Takahashi N, Udagawa N, Suda T. A new member of tumor necrosis factor ligand family, ODF/OPGL/TRANCE/ RANKL, regulates osteoclast differentiation and function. Biochemical and biophysical research communications 1999;256:449-455.

48. Chauhan A, Yadav SS, Dwivedi P, Lal N, Usman K, Khattri S. Correlation of Serum and Salivary Cytokines Level With Clinical Parameters in Metabolic Syndrome With Periodontitis. Journal of clinical laboratory analysis 2016;30:649-655.

49. El-Sharkawy H, Aboelsaad N, Eliwa M, et al. Adjunctive treatment of chronic periodontitis with daily dietary supplementation with omega-3 Fatty acids and low-dose aspirin. Journal of periodontology 2010;81:1635-1643.

50. Cutando A, Lopez-Valverde A, de Diego RG, et al. Effect of topical application of melatonin to the gingiva on salivary osteoprotegerin, RANKL and melatonin levels in patients with diabetes and periodontal disease. Odontology 2014;102:290-296.

51. Jin J, Zhang X, Lu Z, et al. Simvastatin inhibits lipopolysaccharide-induced osteoclastogenesis and reduces alveolar bone loss in experimental periodontal disease. Journal of periodontal research 2014;49:518-526.
52. Katz J, Chaushu G, Sharabi Y. On the association between hypercholesterolemia, cardiovascular disease and severe periodontal disease. Journal of clinical periodontology 2001;28:865-868.

53. Lianhui Y, Meifei L, Zhongyue H, Yunzhi F. [Association between chronic periodontitis and hyperlipidemia: a Metaanalysis based on observational studies]. Hua xi kou qiang yi xue za zhi = Huaxi kouqiang yixue zazhi = West China journal of stomatology 2017;35:419-426.

54. Liao JK. Isoprenoids as mediators of the biological effects of statins. The Journal of clinical investigation 2002;110:285-288.

55. Uzzan B, Cohen R, Nicolas P, Cucherat M, Perret GY. Effects of statins on bone mineral density: a meta-analysis of clinical studies. Bone 2007;40:1581-1587.

56. Miricescu D, Totan A, Calenic B, et al. Salivary biomarkers: relationship between oxidative stress and alveolar bone loss in chronic periodontitis. Acta odontologica Scandinavica 2014;72:42-47.

57. Niederman R. Evidence-based dentistry: what is it, and what does it have to do with practice? The methods of evidence-based dentistry. Quintessence international 1998; 29:811-817. 